\title{
Nonclassical Austenite-Martensite Interfaces
}

\author{
J.M. Ball and C. Carstensen* \\ Mathematical Institute, University of Oxford, 24-29 St Giles, Oxford OXI 3LB, U.K. \\ * Mathematisches Seminar, Christian-Albrechts-Universität zu Kiel, Ludewig-Meyn-Str. 4, 24098 Kiel, \\ Germany
}

\begin{abstract}
In the last 10 years a new theory of martensitic transformations based on nonlinear elasticity has been developed and intensively studied by J.M. Ball \& R.D. James and others. Microstructures are understood in the theory as minimizing sequences for the total free energy, and are conveniently described using Young measures.

For the case of the classical austenite-martensite interface, in which a pure phase of austensite meets a simple laminate of martensite, the formulae of the crystallographic theory of martensite for the orientation of the habit plane are recovered from the energy-minimization picture.

The theory leads naturally to the prediction of more complex austenite-martensite interfaces, in which a pure phase of austenite meets a more complicated microstructure of martensite. The possible interfaces are computed in case that the martensite wells correspond to an orthorombic to monoclinic lattice transformation. Besides the classical interfaces, with simple laminate of martensite, new possible interfaces are presented which correspond for a larger set of lattice parameters. Then, for certain parameters, it can be concluded from the theory that possible interfaces are planes.
\end{abstract}

\section{INTRODUCTION}

The classical austenite-martensite interface in a single crystal is a plane (the habit plane) separating undistorted austenite from a simple laminate consisting of fine twins of martensite. In this paper we investigate theoretically the possibility that the interface separates undistorted austenite from a more complicated microstructure of martensite, such as a double laminate (layers within layers structure). We show, for example, that for a cubic to tetragonal transformation such a nonclassical interface is energetically preferred for certain regions in lattice-parameter space. We also study the question of whether such nonclassical interfaces are planar or could be curved.

The classical crystallographic theory of martensite [8] delivers formulae for the orientation of the habit plane and for the phase fractions of each variant of martensite in the simple laminate. For example, in a cubic to tetragonal transformation there are 24 possible habit-plane normals, 8 corresponding to each pair of the three tetragonal variants, and the theory reveals restrictions on the lattice parameters (see Fig. 2-4 below) for classical austenite-martensite interfaces to exist.

The crystallographic theory can be deduced as a consequence of a geometrically nonlinear theory of martensitic transformations that has been developed in recent years $([3,4])$, in which microstructures are identified with 'minimizing sequences' for the total free energy. This energy is given by

$$
I_{\theta}(y)=\int_{\Omega} \varphi(\nabla y(x), \theta) d x
$$


Here $y(x)$ denotes the deformed position of the particle at $x \in \Omega$, where the reference configuration $\Omega$ represents undistorted austenite. The free-energy function $\varphi(F, \theta)$ depends on the deformation gradient $F$ and the temperature $\theta$. By frame-indifference $\varphi(R F, \theta)=\varphi(F, \theta)$ for all $F, \theta$ and for all rotations $R$, i.e. for all $3 \times 3$ matrices in the set $S O(3):=\left\{R: R^{T} R=1, \operatorname{det} R=1\right\}$. At the transformation temperature $\theta_{c}$ the energy wells of $\varphi$ are given by $S O(3)$ for the austenite, and by $S O(3) U_{i}$ for each of the $N$ different variants of martensite, with $U_{i}=U_{i}^{T}>0$ for each $i$. Thus $\varphi\left(F, \theta_{c}\right) \geq 0$ with $\varphi\left(F, \theta_{c}\right)=0$ precisely for those $F$ in the set

$$
S O(3) \cup \bigcup_{i=1}^{N} S O(3) U_{i}
$$

For $\theta>\theta_{c}, \varphi$ is minimized just at the austenite well $S O(3) \alpha(\theta) 1$, while for $\theta<\theta_{c}$ the martensite wells minimize energy. Here $\alpha(\theta), \alpha\left(\theta_{c}\right)=1$, describes the thermal expansion of the austenite.

For a cubic to tetragonal transformation we have $N=3$ and

$$
U_{1}=\operatorname{diag}\left(\eta_{2}, \eta_{1}, \eta_{1}\right), U_{2}=\operatorname{diag}\left(\eta_{1}, \eta_{2}, \eta_{1}\right), U_{3}=\operatorname{diag}\left(\eta_{1}, \eta_{1}, \eta_{2}\right),
$$

where the lattice parameters $\eta_{1}>0, \eta_{2}>0, \eta_{1} \neq \eta_{2}$. For an orthorhombic to monoclinic transformation, on the other hand, we have $N=2$ and

$$
U_{1}=\operatorname{diag}\left(\eta_{2}, \eta_{1}, \eta_{3}\right), U_{2}=\operatorname{diag}\left(\eta_{1}, \eta_{2}, \eta_{3}\right),
$$

with $\eta_{i}>0, \eta_{1} \neq \eta_{2}([4, \mathrm{p} 403])$.

In order to recover the crystallographic theory of martensite from this model we proceed as follows [3]. We consider a planar austenite-martensite interface $\{x \cdot m=k\}$ and try and choose the habit-plane normal $m$ in such a way that there exists a minimizing sequence $y^{(j)}$ of deformations, i.e. a sequence such that $I_{\theta_{c}}\left(y^{(j)}\right) \rightarrow 0$ as $j \rightarrow \infty$. We require that, for $x \cdot m<k, y^{(j)}$ corresponds to austenite, so that the values of $\nabla y^{(j)}(x)$ tend to $S O(3)$ in an appropriate sense; it can be shown that without loss of generality this amounts to assuming that $\nabla y^{(j)}(x) \rightarrow 1$ for $x \cdot m<k$, except possibly for a set of points $x$ of zero volume. For $x \cdot m>k$ we require that $y^{(j)}$ corresponds to a simple laminate of martensite, and we choose this to have the form that, except in a thin transition layer near the habit plane whose volume tends to zero as $j \rightarrow \infty, \nabla y^{(j)}$ takes only two values $A, B$ belonging to two of the martensite wells, say $A \in S O(3) U_{i}, B \in S O(3) U_{j}, i \neq j$, in alternate layers of thicknesses $\lambda / j,(1-\lambda) / j$ respectively with normal $n$, where $0<\lambda<1$. For geometric compatibility we need that $A, B$ are rank-one connected, i.e.

$$
A-B=a \otimes n
$$

for vectors $a, n$. Said differently, the laminate is twinned. It is shown in [3] that in order for it to be possible to choose $A, B, \lambda, m$ in this way and such that $y^{(j)}$ is a minimizing sequence, it is necessary and sufficient that they be chosen such that the macroscopic deformation gradient (or weak limit) $\nabla y$ corresponding to $\nabla y^{(j)}$ be compatible. For $x \cdot m>k$ this macroscopic deformation gradient is the constant matrix $\lambda A+(1-\lambda) B$, whereas for $x \cdot m<k$ we have $\nabla y(x)=1$. Thus $A, B, \lambda, m$ need to be chosen such that

$$
1-(\lambda A+(1-\lambda) B)=b \otimes m
$$

for some $b$. Analyzing this condition gives rise precisely to the formulae of the crystallographic theory of martensite. In fact this new perspective gives more information, for example that the interface is necessarily planar (see [3] and for other developments $[6],[2]$ ).

\section{NONCLASSICAL PLANAR INTERFACES}

In order to discuss the possibility of nonclassical austenite-martensite interfaces we follow the same general idea, but this time allow $\nabla y^{(j)}$ to represent a general energy-minimizing microstructure of 
martensite rather than restrict attention to the case when this microstructure is a simple laminate. That is we require that for $x \cdot m>k$ we have that $\nabla y^{(j)}(x)$ tends in a suitable sense as $j \rightarrow \infty$ to the set $\mathcal{K}:=\bigcup_{i=1}^{N} S O(3) U_{i}$ consisting of the martensitic energy wells. (To be precise we require that the Young measure $\left(\nu_{x}\right)_{x \in \Omega}$ of $\nabla y^{(j)}$ is supported in $\mathcal{K}$. See [4] for details, which are not needed here.)

We first restrict attention to the case when the martensitic microstructure is homogeneous in the sense that the corresponding macroscopic deformation gradient $\nabla y(x)=F$ is independent of $x$ with $x \cdot m>k$. The set of all such matrices $F$ is called the quasiconvexification of $\mathcal{K}$ and is written $Q(\mathcal{K})$. It can be shown that $Q(\mathcal{K})$ is the same as the set of $F$ such that there exists a sequence $z^{(j)}$ of deformations satisfying $z^{(j)}(x)=F x$ for $x$ belonging to the boundary $\partial \Omega$ of $\Omega$ and such that $\nabla z^{(j)}(x) \rightarrow \mathcal{K}$ in the sense indicated above.

Given that we know $Q(\mathcal{K})$ for a given set of martensitic energy wells, we need only examine whether it is possible to find a rank-one connection between $S O(3)$ and $Q(\mathcal{K})$, i.e. whether there exist vectors $b, m$ such that

$$
1+b \otimes m \in Q(\mathcal{K})
$$

Here we have without loss of generality (since $R Q(\mathcal{K})=Q(\mathcal{K})$ for any $R \in S O(3)$ ) chosen the matrix in the austenite well to be the identity. The vectors $m$ will thus give possible normals for the nonclassical habit plane. Since $Q(\mathcal{K})$ turns out to be a much bigger set than that consisting of all matrices $F=\lambda A+(1-\lambda) B$, where $0 \leq \lambda \leq 1$ and $A \in S O(3) U_{i}, B \in S O(3) U_{j}, i \neq j, A-B=a \otimes n$ for some $a, n$, there are many more possibilities for nonclassical interfaces than for classical ones. In particular, such interfaces can exist for values of the lattice parameters that prohibit the existence of a classical interface.

Unfortunately at present we only know how to calculate $Q(\mathcal{K})$ for the case of two martensitic wells. We thus restrict attention to this case, and let $N=2$ with $U_{1}, U_{2}$ given by (1.4), so that

$$
\mathcal{K}=S O(3) U_{1} \cup S O(3) U_{2}
$$

In this case it is known that

$$
\begin{aligned}
Q(\mathcal{K}) & =S O(3) \mathcal{F} \\
& :=\{R F: R \in S O(3), F \in \mathcal{F}\},
\end{aligned}
$$

where $\mathcal{F}$ consists of those $F$ with $\operatorname{det} F>0$ such that

$$
F^{T} F=\left[\begin{array}{ccc}
a & c & 0 \\
c & b & 0 \\
0 & 0 & \eta_{3}^{2}
\end{array}\right]
$$

where $a, b>0$ and

$$
\begin{gathered}
a b-c^{2}=\eta_{1}^{2} \eta_{2}^{2}, \\
a+b+2|c| \leq \eta_{1}^{2}+\eta_{2}^{2} .
\end{gathered}
$$

A characterization equivalent to this for the case when $\eta_{3}=\eta_{1}$ is given in [4], and more directly in [2]; the general case can be reduced to this one via a simple linear transformation. Any $F \in Q(\mathcal{K})$ can be obtained in particular as the macroscopic deformation gradient corresponding to a double laminate.

We thus need to determine when

$$
1+b \otimes m \in S O(3) F
$$

for some $b, m$ and $F$ of the above form. This is equivalent to asking when the wells $S O(3)$ and $S O(3) F$ are rank-one connected, a question that is answered by the following lemma (used also in analyzing the existence of twins and (1.6)).

Lemma 2.1 ([3]). Let $F$ be a nonsingular matrix that is not a rotation. Then, the wells $S O(3)$ and $S O(3) F$ are rank-one connected if and only if the middle eigenvalue of $F^{T} F$ is one. In this case, $1+b \otimes m \in S O(3) F$ for some $b$ if and only if $m$ is a non-vanishing multiple of one of the two vectors 
$\sqrt{1-\lambda_{1}} e_{1} \pm \sqrt{\lambda_{3}-1} e_{3}$, where $0 \leq \lambda_{1} \leq 1 \leq \lambda_{3}$ are the three eigenvalues of $F^{T} F$ with corresponding orthonormal eigenvectors $e_{1}, e_{2}, e_{3}$.

We assume that $\eta_{3} \neq 1$; the case $\eta_{3}=1$ can be handled similarly (see[1]). Thus for (2.7) to hold one of the eigenvalues of $\left[\begin{array}{ll}a & c \\ c & b\end{array}\right]$ is 1 . Let the other be $\mu$. Hence,

$$
a+b=1+\mu \text { and } \mu=a b-c^{2} .
$$

From (2.5) $\mu=\eta_{1}^{2} \eta_{2}^{2}$ and we have to analyse the restriction (2.6). To do this (cf [2]) introduce the new variables $a-b$ and $a+b=1+\eta_{1}^{2} \eta_{2}^{2}$. We observe that (2.6) is

$$
2|c| \leq \eta_{1}^{2}+\eta_{2}^{2}-1-\eta_{1}^{2} \eta_{2}^{2}=-\left(1-\eta_{1}^{2}\right)\left(1-\eta_{2}^{2}\right)
$$

and note that $\eta_{1}, \eta_{2}<1$ or $\eta_{1}, \eta_{2}>1$ are thus prohibited. Writing $4 a b=(a+b)^{2}-(a-b)^{2}=$ $\left(1+\eta_{1}^{2} \eta_{2}^{2}\right)^{2}-(a-b)^{2}$, we obtain from $(2.5)$ that

$$
(2 c)^{2}+(a-b)^{2}=\left(1-\eta_{1}^{2} \eta_{2}^{2}\right)^{2} .
$$

The geometric interpretation of $(2.9),(2.10)$ is depicted in Fig.1. In the $(a-b, 2 c)$ plane (2.10) is a circle with centre the origin and radius $\left|1-\eta_{1}^{2} \eta_{2}^{2}\right|$. Since we require that 1 is the middle eigenvalue of $F^{T} F$ we deduce that an interface is possible provided that, in the case $\eta_{3}<1$,

$$
\eta_{2}^{-1} \leq \eta_{1} \leq 1 \text { or } 1 \leq \eta_{2}^{-1} \leq \eta_{1},
$$

and in the case $\eta_{3}>1$,

$$
\eta_{2} \leq \eta_{1}^{-1} \leq 1 \text { or } 1 \leq \eta_{2} \leq \eta_{1}^{-1}
$$

For the existence of a classical austenite martensite interface (2.6) is replaced by

$$
a+b+2|c|=\eta_{1}^{2}+\eta_{2}^{2}
$$

and from Fig. 1 we see that this is possible for some $a, b, c$ provided

$$
\eta_{1}^{2}+\eta_{2}^{2}-1-\eta_{1}^{2} \eta_{2}^{2} \leq\left|1-\eta_{1}^{2} \eta_{2}^{2}\right|
$$

Thus for a classical interface we require that $\eta_{1}^{2}+\eta_{2}^{2} \leq 2$ if $\eta_{1} \eta_{2} \leq 1$, and $\eta_{1}^{-2}+\eta_{2}^{-2} \geq 2$ if $\eta_{1} \eta_{2} \geq 1$. These results are displayed in Fig.2, Fig.3 below.

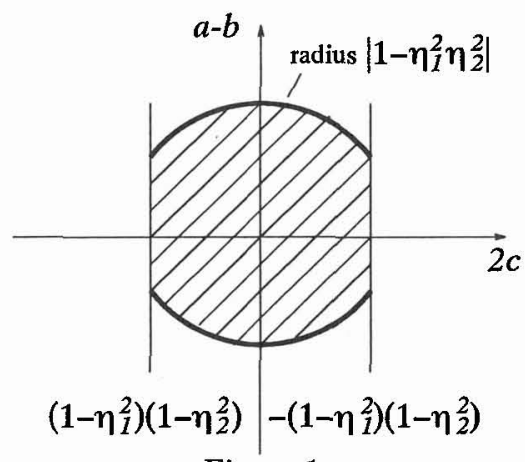

Figure 1:

Restrictions (2.9) and (2.10) on Parameters $(a-b, 2 c)$

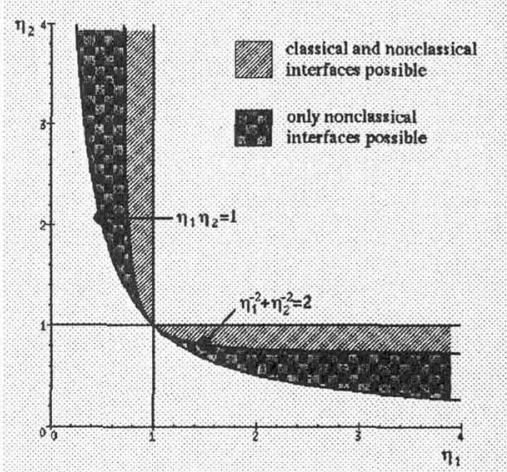

Figure 2:

Parameters $\left(\eta_{1}, \eta_{2}\right)$ with $(2.11)$ Allowing Interfaces in Case $\eta_{3}<1$

The normals $m$ of the possible nonclassical habit planes are deduced from Lemma 2.1. Setting

$$
a-b=\left(1-\eta_{1}^{2} \eta_{2}^{2}\right) \cos \omega, 2 c=\left(1-\eta_{1}^{2} \eta_{2}^{2}\right) \sin \omega,
$$

the results are that, up to an irrelevant constant factor,

$$
m=\left(\left|1-\eta_{1}^{2} \eta_{2}^{2}\right|^{1 / 2} \cos (\omega / 2),-\left|1-\eta_{1}^{2} \eta_{2}^{2}\right|^{1 / 2} \sin (\omega / 2), \pm \eta_{1}\left|1-\eta_{3}^{-2}\right|^{1 / 2}\right) .
$$


In the case when classical interfaces exist, the normals given by (2.16), when normalized to be unit vectors, form 4 circular arcs on the unit sphere, whose end-points are the 8 habit planes of the classical theory corresponding to the variants $U_{1}, U_{2}$. When $\eta_{1}, \eta_{2}$ are such that only nonclassical interfaces exist, the possible normals form two complete circles on the unit sphere with centres on the $e_{3}-$ axis and with radii

$$
r=\left(1+\frac{1-\eta_{3}^{-2}}{\eta_{2}^{2}-\eta_{1}^{-2}}\right)^{-1}
$$

The existence of extra habit planes for the nonclassical interfaces could be an energetic incentive for their creation due to the direction of temperature gradients, even when classical interfaces exist.

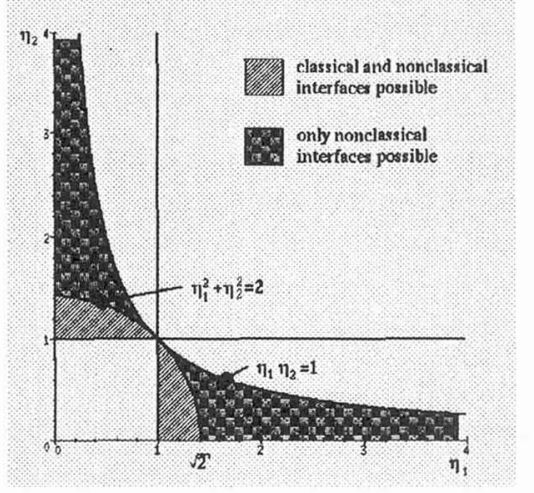

Figure 3:

Parameters $\left(\eta_{1}, \eta_{2}\right)$ with $(2.12)$

Allowing Interfaces in Case $1<\eta_{3}$

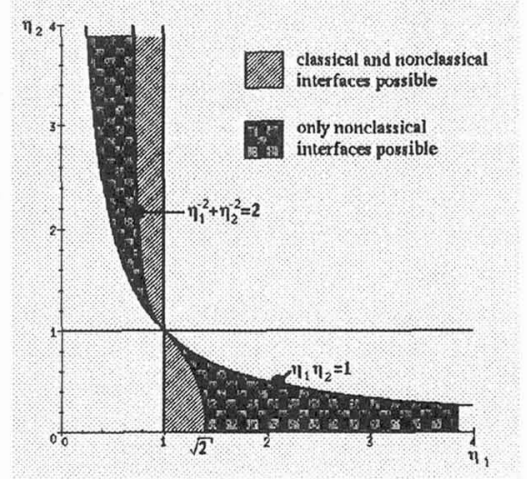

Figure 4:

Parameters $\left(\eta_{1}, \eta_{2}\right)$ Allowing

Interfaces in Case $\eta_{1}=\eta_{3}$

It is interesting to consider the case $\eta_{3}=\eta_{1}$ corresponding to two of the three tetragonal wells in (1.3). In this case the admissible values of $\eta_{1}, \eta_{2}$ for the existence of nonclassical and classical interfaces are obtained by combining the cases $\eta_{3}>1, \eta_{3}<1$ above, and the result is shown in Fig.4. Note that the region in the $\eta_{1}, \eta_{2}$ plane for which only nonclassical interfaces exist has a pair of cusps at $\eta_{1}=\eta_{2}=1$, so that for lattice parameters very near 1 the region corresponding to the existence of a classical interface is much larger. Care would thus be needed to find a material for which the nonclassical interfaces are preferred. It should also be noted that a nonclassical interface might involve greater interfacial energy between variants of martensite than for a simple laminate. Thus even for lattice parameters which according to the above analysis (based on a model that ignores interfacial energy) allow only nonclassical interfaces, the material might still choose a simple laminate with deformation gradients away from the energy wells in preference to a double laminate, say, with gradients on the wells. Whereas double laminates are frequently observed in martensitic transformations, the authors are not aware of any cubic to tetragonal transformations for which a nonclassical interface is known to be responsible for their formation. Nonclassical interfaces have been observed in CuAlNi shape memory alloys [5], for which the transformation is cubic to orthorhombic.

\section{NONHOMOGENEOUS MARTENSITIC MICROSTRUCTURES}

We briefly sketch results that we have obtained for the case when the martensitic microstructure is inhomogeneous, so that the macroscopic deformation gradient depends on $x$ in the region occupied by the martensite. This could happen, for example, if there were a self-similar pattern of microstructure that became finer and finer as an interface is approached. Chu \& James [5] have shown us micrographs 
in which such roughly self-similar microstructures of martensite are seen in CuAlNi alloys, both when the interface separates the martensite from austenite, and when it separates the martensite from another region occupied by martensite. In these micrographs several variants of martensite are involved in the self-similar region.

The first result concerns the possible linear boundary conditions $y(x)=F x$ on a plane $x \cdot m=k$ that are consistent with a martensitic microstructure corresponding to the two wells $\mathcal{K}$ given in (2.2). Equivalently, passing to the weak limit, what are the boundary conditions of the above type that are consistent with a deformation $y$ having $\nabla y(x) \in Q(\mathcal{K})$ for $x \cdot m>k$ ? The answer is that $F+b \otimes m$ must belong to $Q(\mathcal{K})$ for some $b$; that is, the normal $m$ must be consistent with having an interface between a deformation with constant gradient $F$ and a homogeneous martensitic microstructure. The proof relies on a famous theorem of Reshetnyak [7] to the effect that nonconstant quasiregular maps are isolated, the fact that $Q(\mathcal{K})$ is a polyconvex set, and a result proved in [4] to the effect that any $y$ with $\nabla y(x) \in Q(\mathcal{K})$ for $x$ in a bounded domain is a plane strain.

The second result starts from the hypothesis that

$$
\Omega=\Omega_{A} \cup \Gamma \cup \Omega_{M}
$$

for an open austenite domain $\Omega_{A}$, an open martensite domain $\Omega_{M}$ and an interface $\Gamma$ of finite area separating them. Suppose that there is a corresponding zero-energy microstructure, so that passing to the weak limit we have $y$ with $\nabla y(x) \in S O(3)$ for $x \in \Omega_{A}$ and $\nabla y(x) \in Q(\mathcal{K})$ for $x \in \Omega_{M}$. Then if $\eta_{3} \neq 1, \Gamma$ is locally a plane and $\nabla y$ is locally constant on either side of $\Gamma$. This means that for these two martensitic variants curved nonclassical interfaces and self-similar behaviour are impossible. The proof uses the plane strain result from [4] and an integration argument.

Precise statements and details of the proofs of these results, and of those in Section 2 will appear in [1].

\section{References}

[1] J.M. Ball and C. Carstensen. To appear.

[2] J.M. Ball and R.D. Jannes. Untitled book on the mathematics of microstructure. In preparation.

[3] J.M. Ball and R.D. James. Fine phase mixtures as minimizers of energy. Arch. Rat. Mech. Anal., 100:13-52, 1987.

[4] J.M. Ball and R.D. James. Proposed experimental tests of a theory of fine microstructure, and the two-well problem. Phil. Trans. Roy. Soc. London A, 338:389-450, 1992.

[5] C. Chu and R.D. James. Personal communication.

[6] R.D. James and D. Kinderlehrer. theory of diffusionless phase transformations. In D. Serre M. Rascle and M. Slemrod, editors, Partial Differential Equations and Continuum Models of Phase Transitions, pages 51-84. SpringerVerlag, 1989.

[7] Yu. G. Reshetnyak. Space mappings of bounded distortion, volume 73 of Translations of Mathematical Monographs. American Mathernatical Society, Providence, 1989.

[8] M.S. Wechsler, D.S. Lieberman, and T.A. Read. On the theory of the formation of martensite. Trans. AIME J. Metals, 197:1503-1515, 1953. 